\title{
Clinical Efficacy Study of the Quadrant Channel and Delta Large Channel Technique in the Treatment of Lumbar Degenerative Diseases
}

\author{
Jia-Jun Zhang' \\ Chuan-Li Zhou' \\ Chong Sun' \\ De-Rong $\mathrm{Xu}^{\prime}$ \\ Mei Bao ${ }^{2}$ \\ Yong Liu'
}

'Department of Spine Surgery, The Affiliated Hospital of Qingdao University, Qingdao, 266003, Shandong, People's Republic of China; ${ }^{2}$ Department of

Pediatric Surgery, The Affiliated Hospital of Qingdao University, Qingdao, 266003, Shandong, People's Republic of China
Correspondence: Yong Liu

Department of Spine Surgery, The

Affiliated Hospital of Qingdao University,

Qingdao, 266003, People's Republic of

China

Tel +86 I3953296687

Email liuyongdr20@163.com
Objective: To compare the therapeutic effect of the quadrant channel and delta large channel techniques in lumbar degenerative diseases.

Methods: According to the inclusion criteria, 62 patients suffering from lumbar degenerative disease were selected for the present study, which was conducted from September 2018 to June 2020. Patients were divided into Group A (quadrant channel technology) and Group B (delta large channel technology), which comprised 28 and 34 patients, respectively. The factors compared between the two groups were operation time, length of incision, blood loss, ambulation time, length of hospitalization, visual analogue scale (VAS) preoperatively, 7 days postoperatively, and 30 days postoperatively, and Oswestry dysfunction score (ODI).

Results: The length of incision, blood loss, ambulation time, and length of hospitalization stay in Group A were significantly higher compared with Group B $(\mathrm{P}<0.05)$. There was no significant difference between the two groups in operation time, preoperative ODI index, preoperative VAS scores, and thirty-day postoperative VAS scores $(\mathrm{P}>0.05)$. The seven-day postoperative VAS score, seven-day postoperative ODI index, and thirty-day postoperative ODI index of Group A were significantly higher than those of Group B $(\mathrm{P}<0.05)$. The preoperative VAS score and ODI index in both groups were significantly higher compared with after operation $(\mathrm{P}<0.05)$.

Conclusion: Both surgical methods achieved a good clinical outcome in the treatment of lumbar degenerative diseases. The delta large channel technique may offer some advantages over quadrant channel technology, such as less trauma and bleeding and faster recovery time.

Keywords: quadrant channel, delta large channel technique, lumbar degenerative diseases

\section{Introduction}

Lumbar degenerative diseases (LDDs) include lumbar disc herniation, lumbar spinal stenosis, lumbar spondylolisthesis, lumbar scoliosis, and spinal instability. According to the literature, around half the elderly population suffers from LDDs. ${ }^{1}$ The method of surgery for treating LDD has gradually changed from traditional open surgery to minimally invasive surgery. In recent years, minimally invasive technology has become a research hotspot in various clinical fields. Percutaneous transforaminal endoscopic discectomy (PTED) was originally conducted as a treatment for lumbar disc herniation. However, given increasing expectations for minimally invasive surgery, PTED is not appropriate for treating all types of lumbar disc herniation and lumbar spinal stenosis. The Interlaminar Endoscopic Surgical System (iLESSYS) has 
emerged as an alternative to PTED. Compared with traditional endoscopy, it has a larger working tube and can more readily treat certain types of lumbar disc herniation and lumbar spinal stenosis. The quadrant channel through the intermuscular space to the lamina uses an "opening the small window" approach to treating LDD. Both Delta large channel and Quadrant channel technologies have their respective indications, but the indications for both have many similarities, and how we should make a better choice between the two when faced with a patient whose indications are consistent with both, was the aim of our present study. The present study retrospectively analyzed and compared the clinical data of 62 patients who underwent LDD surgery in the Affiliated Hospital of Qingdao University from September 2018 to June 2020, including 28 cases of Quadrant channel and 34 cases of Delta large channel surgery. The clinical efficacy of the two techniques in the treatment of LDD was compared.

\section{Materials and Methods}

\section{Patients}

Patient characteristics: Sixty-two patients admitted to hospital due to LDD were selected as observation subjects. Patients were divided into Group A (Quadrant channel technology) and Group B (Delta large channel technology), comprising 28 patients and 34 patients, respectively. The general conditions of patients in the two groups are shown in Table 1. There was no statistically significant difference between the two groups of patients in terms of general information, such as age, gender, course of disease, and diseased segments $(\mathrm{P}>0.05)$. Inclusion Criteria: (1) The patient had low back pain or radiating pain in the lower extremities; (2) imaging examination consistent with the characteristics of single segment spinal canal stenosis with disc herniation; (3) three months of bed rest, hot compress, physiotherapy, and other conservative treatment were ineffective; (4) no contraindications of operation; (5) willing to comply with regular follow-ups; and (6) the patients and their family members understood the operation method and signed the informed consent. Exclusion criteria: (1) Spinal tumors, tuberculosis, and other infectious diseases; (2) patients with a history of lumbar spine surgery or fracture; (3) local infection or skin injury of the incision area; (4) multisegmental degeneration of the lumbar spine with symptoms; and (5) imaging indicating lumbar spondylolisthesis and lumbar instability, requiring fusion surgery.

\section{Operative Technique}

Group A: The patients were put in a prone position, $2.5 \%$ iodophor was used for routine disinfection, and a surgical towel was draped routinely. After positioning under fluoroscopy, a median longitudinal incision of around $3 \mathrm{~cm}$ in length was made beside the lesion space on the affected side. The skin, subcutaneous tissue, and lumbar fascia were cut in turn, and the paravertebral muscles were separated with a Quadrant instrument to establish the channel. On the affected side of the lesion segment, the lower edge of the upper lamina and the upper edge of the lower lamina were exposed. An electric grinder was used to remove part of the lower edge of the upper lamina on the affected side of the lesion segment and "open the window." Exploration revealed obvious hypertrophy of the yellow ligament, partial adhesion to the dura, and stenosis of the central spinal canal. The hypertrophic yellow ligament was excised, and the central spinal canal was decompressed. The disc herniation in the lesion segment was observed, which was compressing the nerve root on the affected side. The protruding nucleus pulposus tissue was removed, and the nerve root on the affected side was fully decompressed. The incision was rinsed with normal saline, a drainage tube was placed after counting the gauze and the instrument, and the incision was sutured layer by layer, as shown in Figure 1.

Table I General Information of Patients in Both Groups

\begin{tabular}{|l|l|l|l|l|}
\hline Clinical Information & Group A (28 Cases) & Group B (34 Cases) & t/ $\chi^{2}$ & -value \\
\hline Gender (male/female) & $13 / 15$ & $14 / 20$ & 0.172 & 0.798 \\
\hline Average age (year) & $54.9 \pm 14.5$ & $61.3 \pm 14.4$ & -1.737 & 0.088 \\
\hline Average course (month) & $41.2 \pm 88.3$ & $31.5 \pm 35.1$ & 0.546 & 0.589 \\
\hline $\begin{array}{l}\text { Diseased segment } \\
\text { L3/4 } \\
\text { L4/5 } \\
\text { L5/SI }\end{array}$ & 3 & 1 & 2.681 & 0.262 \\
\hline
\end{tabular}




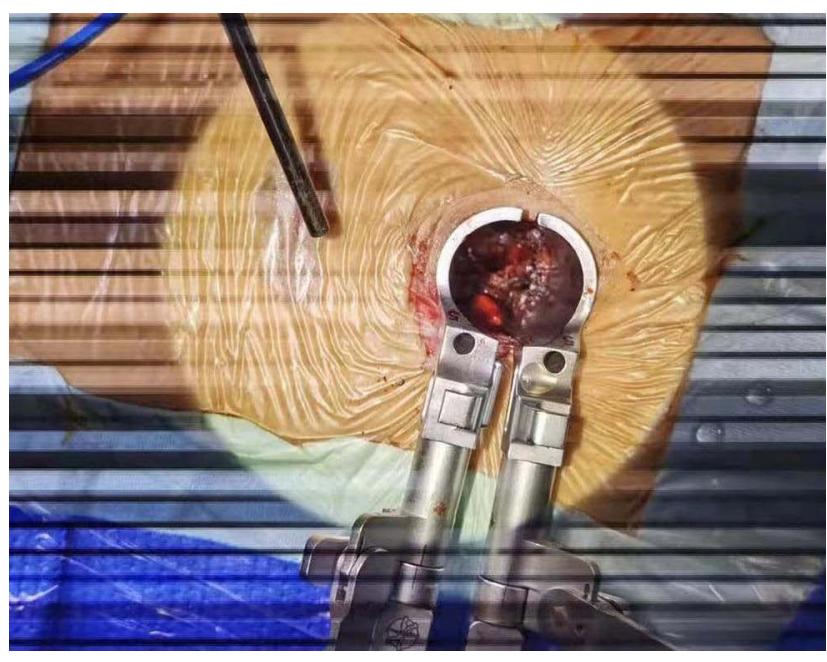

Figure I Quadrant channel.

Group B: The patient was placed in the prone position, the chest and iliac parts were raised, and the affected vertebral plate space of the lesion segment was located under fluoroscopy. The appropriate position of the upper external edge of the vertebral plate space was used as the puncture point. The skin was routinely disinfected, a sterilized cave towel was laid, and the lesions were punctured to the outer upper margin of the affected side lamina space under anterior and lateral fluoroscopy. A guide wire was inserted, and the skin was cut at the insertion point with a diameter of around $1 \mathrm{~cm}$. The guide rod was placed along the guide wire, and the cannula was expanded step by step before finally placing the working cannula. The $\mathrm{C}$-arm was used to confirm that the working sleeve was in a good position, was the guide wire and guide rod, the intervertebral foraminal mirror was connected and flushing with normal saline was conducted. The intervertebral foraminal lens was placed into the working casing, and the appropriate water flow rate was adjusted. After the muscle, fat, and other soft tissues in the visual field were bitten off, and the upper and lower vertebral plates and facet joints were identified, the hypertrophic yellow ligament was bitten off directly; the lateral recess of the decompression side, dural sac, and nerve root were exposed; and the bleeding was stopped with radio frequency. The protrusion of the herniated disc at the diseased segment was observed, the nucleus pulposus was removed slowly with grasping forceps, and the intervertebral space was cleared. The lower nerve root was relaxed, the dural sac expanded satisfactorily, and no nucleus pulposus remained in the spinal canal. The working sleeve was removed, and the wound was sewn up, as shown in Figure 2.

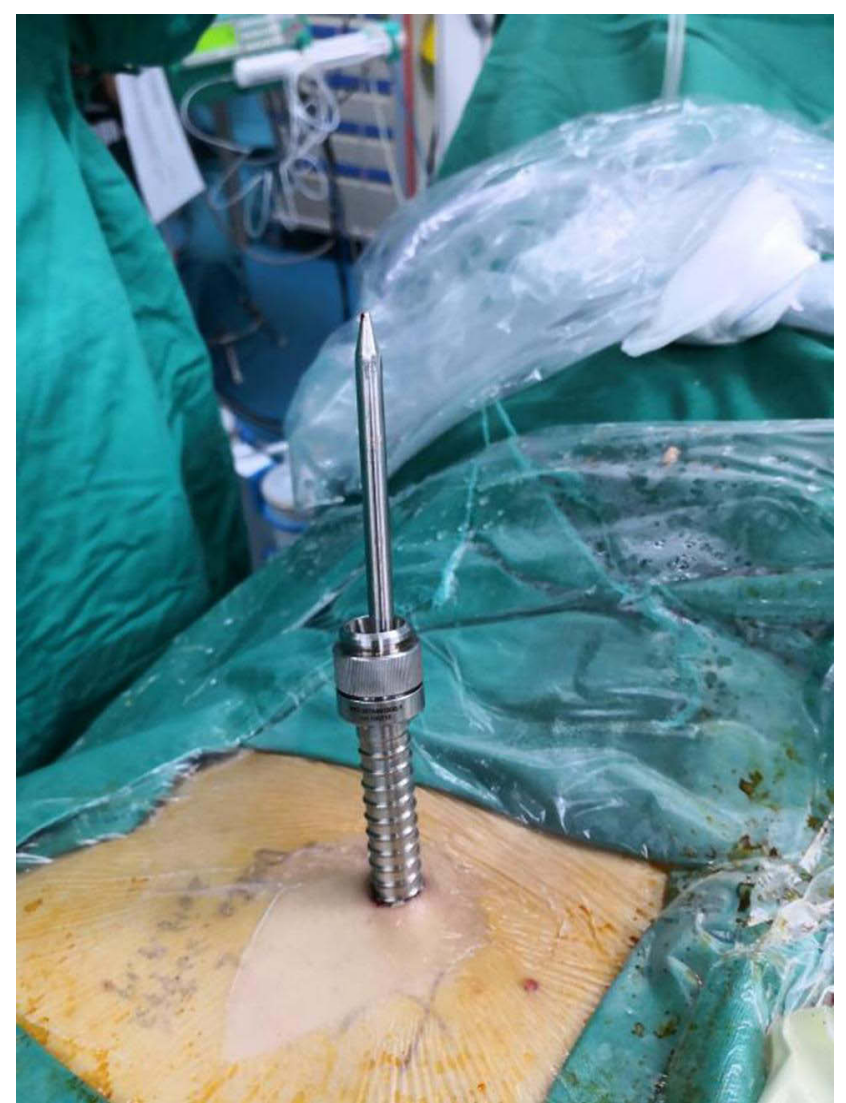

Figure 2 Delta large channel.

\section{The Evaluation Index}

The length of incision, operation time, blood loss, ambulation time, and length of hospitalization of the two groups were counted. The VAS score and ODI index of the two groups were recorded.

\section{Statistical Method}

SPSS 26.0 software was used for the statistical data analysis. The normally distributed data were expressed as mean \pm standard deviation $(\mathrm{x} \pm \mathrm{s})$. Comparisons of measurement data were made by $t$-test, comparisons of count data between two groups were made by $\chi^{2}$ test, and comparisons of VAS scores and ODI indexes between two groups were made by ANOVA with repeated measures design. $\mathrm{P}<0.05$ indicated that the difference between the data was statistically significant.

\section{Results}

\section{General Conditions in the Perioperative Period}

There was no significant difference in operation time between the two groups $(\mathrm{P}>0.05)$. The length of incision, 
Table 2 The General Situation of the Two Groups of Patients During Perioperative Period

\begin{tabular}{|l|l|l|l|l|l|}
\hline Group & $\begin{array}{l}\text { The Operation } \\
\text { Time (Min) }\end{array}$ & $\begin{array}{l}\text { Length of } \\
\text { Incision } \mathbf{( c m )}\end{array}$ & Blood Loss $(\mathbf{m L})$ & Ambulation Time (d) & $\begin{array}{l}\text { Length of } \\
\text { Hospitalization }(\mathbf{d})\end{array}$ \\
\hline A & $97.0 \pm 21.7$ & $3.0 \pm 0.1$ & $22.3 \pm 18.9$ & $1.8 \pm 0.4$ & $7.4 \pm 2.4$ \\
\hline B & $104.9 \pm 22.7$ & $1.0 \pm 0.1$ & $11.2 \pm 5.9$ & $1.1 \pm 0.3$ & $5.6 \pm 1.9$ \\
\hline t-value & 1.406 & 75.652 & 3.005 & 6.897 & 3.251 \\
\hline$p$-value & 0.165 & 0.000 & 0.005 & 0.000 & 0.002 \\
\hline
\end{tabular}

blood loss, ambulation time, and length of hospitalization stay in group A were significantly higher compared with group $\mathrm{B}(\mathrm{P}<0.05)$, as shown in Table 2 .

\section{Comparison of VAS Score and ODI Index}

There was no significant difference between the two groups in preoperative ODI index, preoperative VAS scores, and thirty-day postoperative VAS scores $(\mathrm{P}>0.05)$. The 30-day postoperative ODI index, seven-day postoperative VAS score, and ODI index of group A were significantly compared with group $\mathrm{B}(\mathrm{P}<0.05)$, as shown in Table 3 . The postoperative VAS score and ODI index in each group were significantly lower compared with before the operation $(\mathrm{P}<$ $0.05)$, as shown in Table 4.

\section{Typical Case I}

A female patient aged 49 years. Chief complaint: low back pain for two weeks, aggravated with right lower limb pain and numbness for one day. Diagnosis: lumbar disc herniation; lumbar spinal stenosis. Surgical approach: Minimally invasive posterior lumbar spine removal L4-5 nucleus pulposus and spinal canal decompression under Quadrant channel. Imaging data before and after the operation are shown in Figures 3 and 4.

Table 3 Comparison of VAS Score and ODI Index Between the Two Groups

\begin{tabular}{|l|c|c|c|c|c|c|c|}
\hline \multirow{2}{*}{ Group } & \multirow{2}{*}{ Case } & \multicolumn{3}{|c|}{ VAS Score } & \multicolumn{2}{c|}{ ODI Index } \\
\cline { 3 - 8 } & & $\begin{array}{c}\text { Before } \\
\text { Operation }\end{array}$ & $\begin{array}{c}\text { 7 Days After } \\
\text { Operation }\end{array}$ & $\begin{array}{c}\text { 30 Days After } \\
\text { Operation }\end{array}$ & $\begin{array}{c}\text { Before } \\
\text { Operation }\end{array}$ & $\begin{array}{c}\text { 7 Days After } \\
\text { Operation }\end{array}$ & $\begin{array}{c}\text { 30 Days After } \\
\text { Operation }\end{array}$ \\
\hline A & 28 & $6.6 \pm 1.3$ & $3.4 \pm 0.8$ & $1.9 \pm 0.8$ & $49.4 \pm 12.7$ & $16.4 \pm 4.3$ & $10.8 \pm 3.1$ \\
\hline B & 34 & $7.1 \pm 1.5$ & $2.9 \pm 0.9$ & $2.0 \pm 0.7$ & $47.6 \pm 10.7$ & $13.0 \pm 4.4$ & $8.4 \pm 2.5$ \\
\hline F-value & & 1.613 & 5.431 & 0.165 & 0.383 & 9.057 & 11.396 \\
\hline p-value & & 0.209 & 0.023 & 0.686 & 0.539 & 0.004 & 0.001 \\
\hline
\end{tabular}

Table 4 Comparison of VAS Score and ODI Index of Patients in Each Group Before and After Surgery

\begin{tabular}{|l|c|c|c|c|c|c|c|}
\hline \multirow{2}{*}{ Group } & \multirow{2}{*}{ Case } & \multicolumn{3}{|c|}{ VAS Score } & \multicolumn{2}{c|}{ ODI Index } \\
\cline { 3 - 8 } & & $\begin{array}{c}\text { Before } \\
\text { Operation }\end{array}$ & $\begin{array}{c}\text { 7 Days After } \\
\text { Operation }\end{array}$ & $\begin{array}{c}30 \text { Days After } \\
\text { Operation }\end{array}$ & $\begin{array}{c}\text { Before } \\
\text { Operation }\end{array}$ & $\begin{array}{c}\text { 7 Days After } \\
\text { Operation }\end{array}$ & $\begin{array}{c}30 \text { Days After } \\
\text { Operation }\end{array}$ \\
\hline $\mathrm{A}$ & 28 & $6.6 \pm 1.3$ & $3.4 \pm 0.8^{*}$ & $1.9 \pm 0.8^{* \#}$ & $49.4 \pm 12.7$ & $16.4 \pm 4.3^{*}$ & $10.8 \pm 3.1^{* \#}$ \\
\hline $\mathrm{F}^{\mathrm{A}}$ & & & 190.811 & & & 211.042 & $13.0 \pm 4.4^{*}$ \\
\hline $\mathrm{B}$ & 34 & $7.1 \pm 1.5$ & $2.9 \pm 0.9^{*}$ & $2.0 \pm 0.7^{* \#}$ & $47.6 \pm 10.7$ & $8.4 \pm 2.5^{* \#}$ \\
\hline $\mathrm{F}^{\mathrm{B}}$ & & & 254.551 & & & 274.207 & \\
\hline
\end{tabular}

Notes: Compared with before surgery, ${ }^{* P}<0.05$; Compared with 7 days after surgery, ${ }^{\sharp} P<0.05$. 

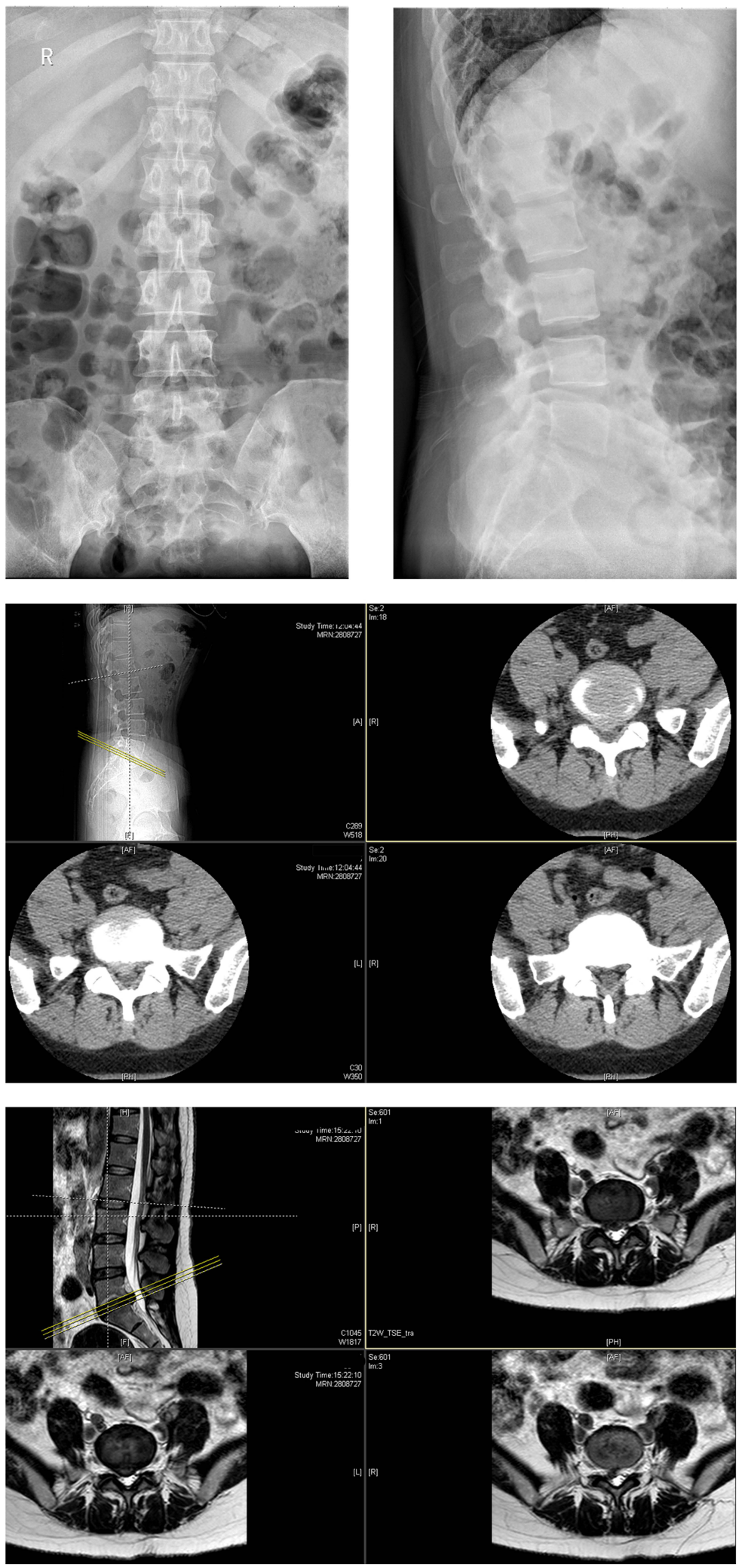

Figure 3 Preoperative X-ray positive and lateral radiographs showed lumbar degeneration. Preoperative CT and MRI showed L5/SI disc herniation and spinal stenosis. 


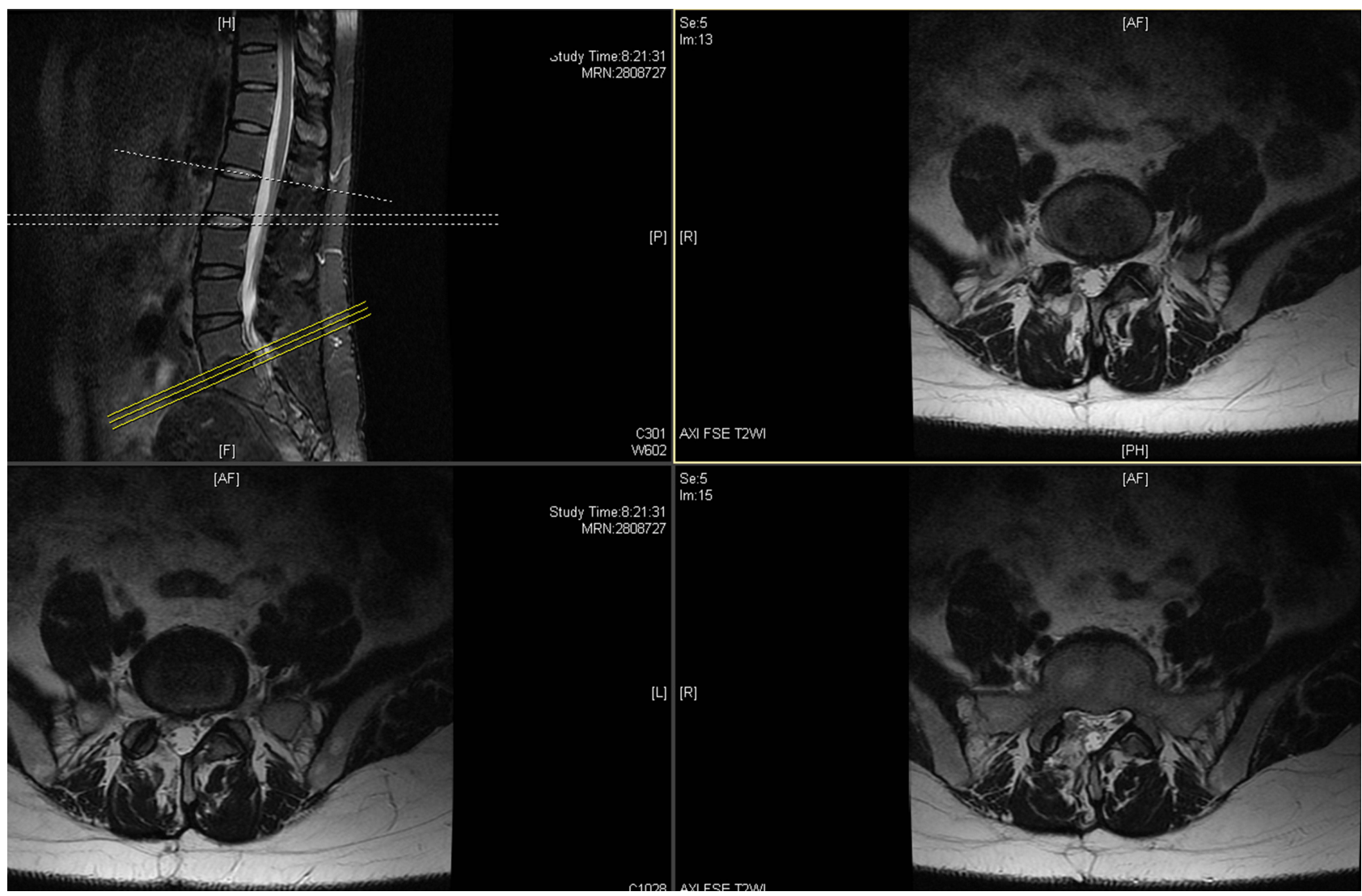

Figure 4 Postoperative MRI showed that the herniated disc disappeared and the spinal canal was enlarged.

\section{Typical Case 2}

A female patient aged 52 years. Chief complaint: pain and weakness in both lower extremities for six months. Diagnosis: lumbar disc herniation; lumbar spinal stenosis. Surgical approach: L4/5 bilateral spinal canal decompression and minimally invasive discectomy under Delta channel. Imaging data before and after the operation are shown in Figures 5 and 6.

\section{Discussion}

LDD is a common disease treated with spinal surgery. The disease harms the patient's physical and mental health and represents a serious economic burden to society and individuals. ${ }^{2,3}$ LDD can be improved after conservative treatment, but any conservative treatment cannot fundamentally solve the problem. ${ }^{4}$ Lumbar spinal stenosis and intervertebral disc herniation were decompressed thoroughly, and then the compression of the nerve root was relieved to achieve the purpose of clinical treatment. Traditional open lumbar surgery leads to a large amount of blood loss and significant trauma and has a long recovery time. Postoperative low back pain is often caused by scar healing of the lower back muscles and can inadvertently lead to iatrogenic spinal instability, requiring an additional surgical operation to stabilize the spine..$^{5-8}$ In recent years, minimally invasive surgery has become a trend, and minimally invasive spinal surgery has been increasingly widely practiced. Both PTED and Quadrant channel have achieved similar results to open surgery. ${ }^{9,10}$ In addition, these techniques can reduce trauma; shorten operation time, length of hospital stay, and rework time; reduce postoperative analgesia demand; reduce infection rate; and decrease the cerebrospinal fluid leakage rate. These techniques are particularly advantageous for middle-aged and elderly populations who have multiple chronic diseases. ${ }^{11,12}$ But so far, there is no relevant research on which of Delta channel technology and Quadrant channel technology developed from PTED can bring better therapeutic effect for patients.

The Quadrant channel, for which surgical incisions are usually around $3 \mathrm{~cm}$ long, creates a working passage via successive stages of expansion of the working trocar, without the need for extensive dissection of paraspinal muscles to reveal bone markers. ${ }^{13-15}$ The incision pain experienced 

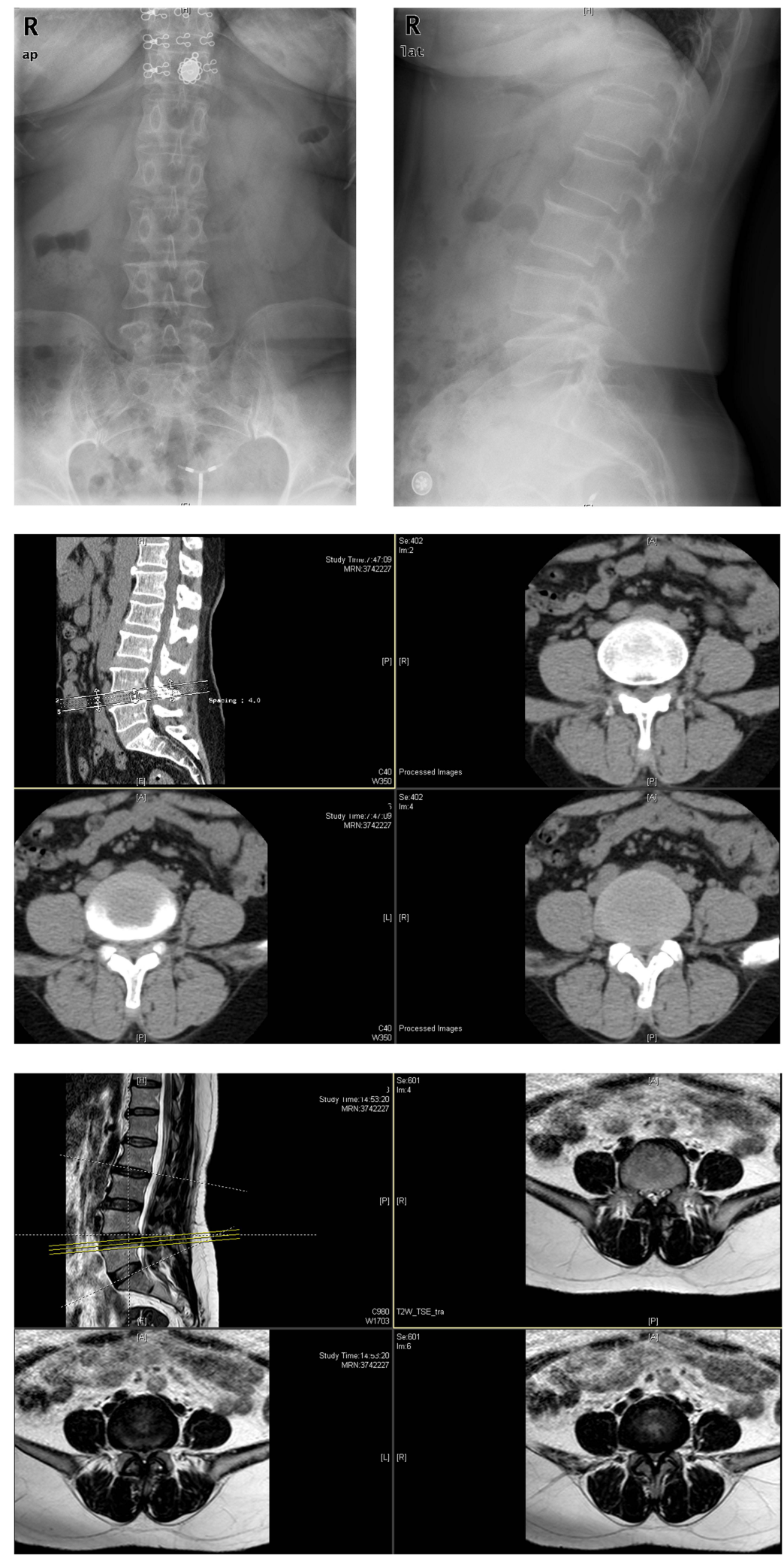

Figure 5 Preoperative X-ray positive and lateral radiographs showed lumbar degeneration. Preoperative CT and MRI showed L4/5 disc herniation and spinal stenosis. 


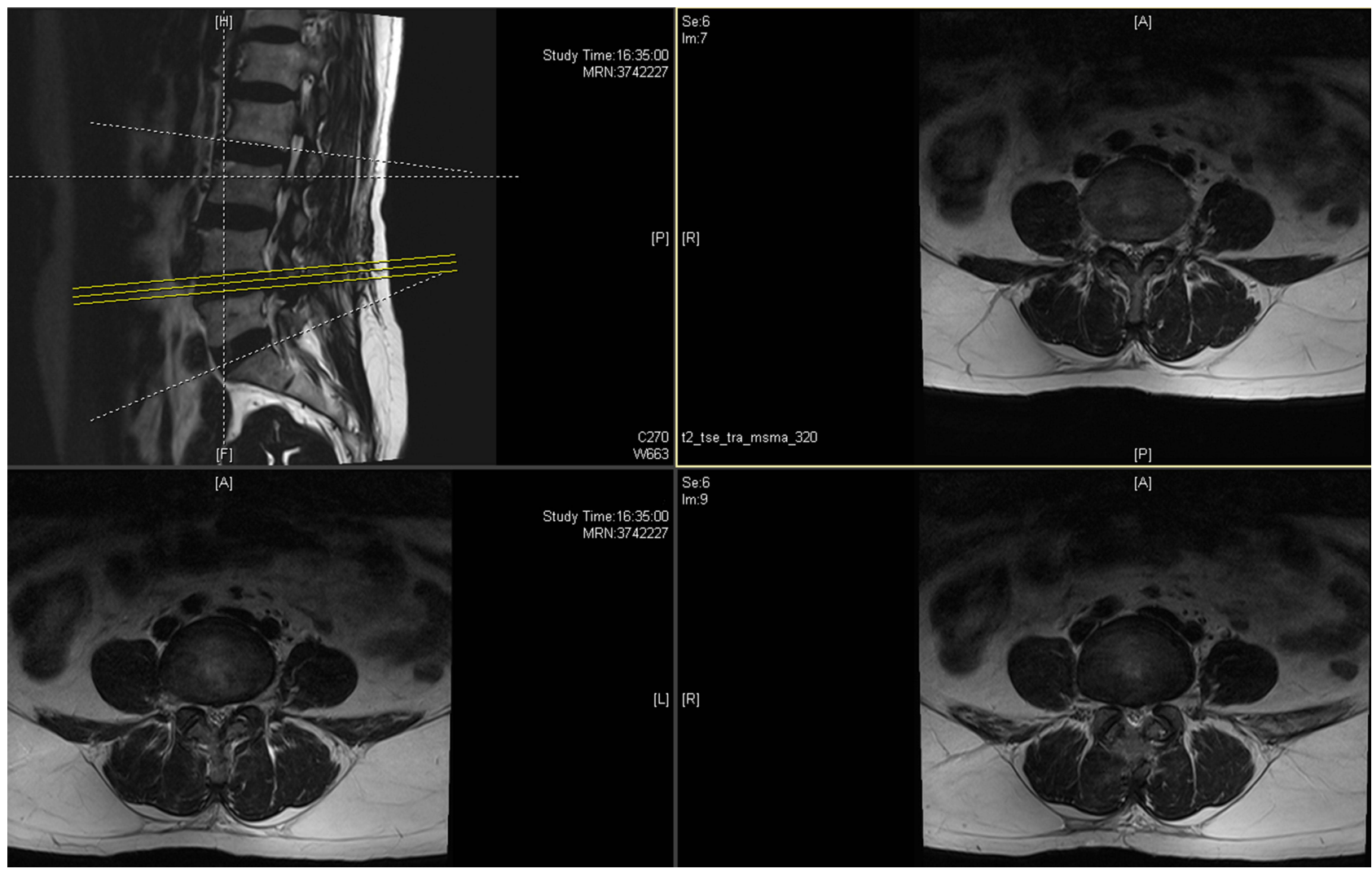

Figure 6 Postoperative MRI showed that the spinal canal was widened and the herniated part of the intervertebral disc vanished.

by patients is relatively mild, and decompression reaches the spinal canal through the interlaminar fenestration, which does not destroy the superior spinous ligament or the interspinous ligament and reduces damage to the vertebral plate, articular process, and other bony structures. The operation can be conducted under direct vision, including the spinal canal decompression, nerve root canal decompression, protruding nucleus pulposus discectomy, and other surgical operations, which avoids eye and hand separation under the microscope and reduces the difficulty of the operation. In addition, many operative procedures are similar to traditional open surgery, which is conducive to rapid mastery. ${ }^{14}$ There were also some drawbacks to this surgical method. The herniated disc must be exposed intraoperatively by pulling the dural sac and nerve root, and there is a risk of nerve root damage and dural tear. ${ }^{16}$ Further, restricted by the limited visual field, hemorrhage of the intraspinal venous plexus is not easily controlled. If spinal dura mater rupture occurs during operation, cerebrospinal fluid outflow combined with venous bleeding can present significant challenges.

Nowadays, the treatment of LDD is developing towards the direction of minimally invasive, and percutaneous transforaminal endoscopic technique is increasingly popular in the treatment of LDD. ${ }^{17}$ Surgical excision of the nucleus pulposus under a foramina lens can be conducted with the Ye-ung approach, the TESSYS approach, and the interlaminar approach. At present, TESSYS technology has become the mainstream minimally invasive treatment of lumbar degenerative diseases, and on this basis, the puncture site, instruments and decompression methods have been improved, and thus derived a new technical route. ${ }^{18,19}$ For some patients with L5/S1 protrusion of intervertebral disc, a surgical approach through the intervertebral foramen is somewhat difficult due to the high anatomical structure of the iliac crest, resulting in the intervertebral disc approach. As this technology has developed, the interlaminar approach is no longer limited to the treatment of the L5/ S1 intervertebral space. iLESSYS evolved from percutaneous endoscopic interlaminar discectomy (PEID), which is a type of PTED. The main indication for PEID is the management of lumbar disc herniation, but its role in lumbar spinal stenosis, particularly in degenerative diseases of the lumbar spine with bone stenosis, is extremely limited due to the small diameter of its working casing. ${ }^{20}$ Delta large channel endoscopy system not only expands the 
channel, but also is equipped with larger grinding drill, which is enough to easily remove redundant bone and hyperplastic articular process. ${ }^{21}$ The report of $\mathrm{Wu}$ et $\mathrm{al}^{22}$ shows that delta system is an effective minimally invasive technique for the treatment of LDD compared with open surgery. Delta large channel technique is a safe and effective minimally invasive operation. In the treatment of lumbar spinal stenosis, this operation can not only relieve the bone compression around the dural sac and nerve root, but also relieve the compression of intervertebral disc, posterior longitudinal ligament and ligamentum flavum and other soft tissues, without obvious damage to the facet joints and joint capsule. Delta large channel technology not only expands the working channel but also has the advantage of percutaneous spinal endoscopy. (1) The working cannula expands step by step to protect as much muscle and soft tissue as possible, avoiding postoperative scar adhesion caused by excessive pulling of paravertebral muscles. Even after recurrence, the difficulty coefficient of the second operation is significantly lower than that of the Quadrant channel. ${ }^{23}$ Only the ligamentum flavum of the hypertrophic part is opened, and the integrity of the posterior column is maintained as much as possible. (2) The operation can be performed under local anesthesia, which can effectively avoid the risks and complications of general anesthesia. Moreover, by communication with the patient, the occurrence of nerve root injury can be detected and avoided in the first instance, opening the era of local anesthesia surgery for lumbar degenerative diseases. ${ }^{24}$ (3) Intraoperative saline was continuously used to infuse the wound to clear the field of vision and enable the important structures to be determined and exposed during the operation. The procedure also has some drawbacks. There is a possibility of recurrence, the rate of which is around $3 \%-5 \%$. Intraoperatively, multiple fluoroscopy is required during cannula placement, and the patient receives a larger dose of radiation. The Delta large channel technique is an eye-hand separation operation that has a steep learning curve and is not easily mastered, which hinders its clinical promotion. ${ }^{25}$ Many scholars have found that for those who received percutaneous spinal endoscopic treatment, the symptoms were improved to a certain extent after giving certain intervention measures. ${ }^{26}$

The surgical incision length in the delta large channel is approximately $1 \mathrm{~cm}$, which is less traumatic and more consistent with the idea of being minimally invasive compared to the quadrant channel. Both procedures reduce the level of postoperative pain and the amount of intraoperative blood loss and accelerate postoperative rehabilitation compared with open surgery because of the smaller surgical incision and less damage to tissues. But for the purposes of this study, the Delta large channel is more effective than quadrant in reducing postoperative pain and intraoperative blood loss and accelerating postoperative rehabilitation. The channel harvested better results due to its smaller surgical incision, less destruction to tissue structure, continuous water perfusion intraoperatively enables various inflammatory mediators as well as byproducts left by electrocoagulation to be washed out, alleviates postoperative surgical incision pain, enables patients to recover as early as possible, and the water pressure formed by continuous perfusion reduces intraoperative bleeding while it is easier to locate the bleeding point and administer blood manipulation. ${ }^{27}$ Firstly, these characteristics of the Delta large channel technology accelerate the postoperative recovery of patients as demonstrated in this study, who had a shorter bed rest and hospital stay, are also in accordance with the current concept of rapid rehabilitation popular in the medical community. Secondly, in terms of short-term efficacy, the delta large channel technique achieved better results and improved patient satisfaction. Although this study did not obtain a comparison of the long-term outcomes of the two procedures because of insufficient follow-up time.

There is no significant difference in the surgical indications between the two surgical methods, though Delta large channel technology may not be able to completely remove the free nucleus pulposus in patients whose free nucleus pulposus position is significantly deviated. Some patients have poor cardiopulmonary function or cerebrovascular status and high surgical risk and cannot tolerate general anesthesia. Delta large channel technology under local anesthesia can be performed for these patients. This is also a key difference between the two surgical indications. The surgeon prioritizes selection based on the surgical indication when choosing which surgical method to use. When two or more surgical methods are applicable to the patient's condition, the patient and their family members have the right to choose the surgical method after listening to the surgeon's explanation of the advantages and disadvantages of various surgical methods. For example, in cases 1 and 2 discussed in this article, the conditions of the two patients were applicable to both surgical procedures, and each patient made different choices and achieved good postoperative results. Delta large channel surgery can be performed only under both local anesthesia and general anesthesia. In the present study, all patients selected in group B were operated on under general anesthesia to avoid the influence of different anesthesia methods on the surgical results of the two groups. In the present study, the amount of intraoperative blood loss in group A was 
significantly higher than that in group B. As the patients in group B did not have a drainage tube after surgery, most patients in group A did not have a drainage tube; therefore, the present study did not include two groups of patients. Detailed comparison and analysis of differences in postoperative blood loss. In the present study, there was no significant difference in operation time between the two groups $(\mathrm{P}>0.05)$. Incision length, blood loss, ambulation time, and length of hospitalization stay in the Quadrant channel group were significantly higher than those in group Delta large channel $(\mathrm{P}<$ 0.05). There was no significant difference between the two groups in preoperative ODI index, preoperative VAS scores, and thirty-day postoperative VAS scores $(\mathrm{P}>0.05)$. The $30-$ day postoperative ODI index, seven-day postoperative VAS score, and ODI index of group A were significantly higher compared with group $\mathrm{B}(\mathrm{P}<0.05)$. The preoperative VAS score and ODI index in both groups were significantly greater compared with after operation $(\mathrm{P}<0.05)$. However, this research does have certain limitations, such as insufficient follow-up time. It can be concluded that Delta Large channel surgery offers the benefits of lower trauma and quicker recovery than Quadrant channel; further, in the present research, the short-term postoperative efficacy was generally superior to the Quadrant channel technique.

\section{Conclusion}

In sum, Delta large channel and Quadrant channel technology each have their own advantages and disadvantages in the treatment of LDD. Both surgical methods can achieve good surgical results. But for this study, the delta large channel technology is more advantageous and can lead to better treatment outcomes for patients. Meanwhile, surgeons should strictly follow the operation indications and choose the appropriate surgical method according to their technical conditions, the patient's symptoms and signs, and imaging findings.

\section{Ethics Approval and Consent to Participate}

The study was conducted in accordance with the Declaration of Helsinki (as was revised in 2013). The study was approved by Ethics Committee of the Affiliated Hospital of the Qingdao University. A written consent was obtained from each patient.

\section{Acknowledgments}

We are particularly grateful to all the people who have given us help on our article.

\section{Funding}

No external funding received to conduct this study.

\section{Disclosure}

The authors declare that they have no competing interests.

\section{References}

1. Bressler HB, Keyes WJ, Rochon PA, Badley E. The prevalence of low back pain in the elderly: a systematic review of the literature. Spine. 1999;24(17):1813-1819. doi:10.1097/00007632-19990901000011

2. Ala-Kokko L. Genetic risk factors for lumbar disc disease. Ann Med. 2002;34(1):42-47. doi:10.1080/078538902317338634

3. Ohashi M, Watanabe K, Hirano T, et al. Predicting factors at skeletal maturity for curve progression and low back pain in adult patients treated nonoperatively for adolescent idiopathic scoliosis with thoracolumbar/lumbar curves: a mean 25-year follow-up. Spine. 2018;43 (23):E1403-E1411. doi:10.1097/BRS.0000000000002716

4. Beyer F, Geier F, Bredow J, et al. Non-operative treatment of lumbar spinal stenosis. Technol Health Care. 2016;24(4):551-557. doi:10.3233/THC-161139

5. Bassewitz H, Herkowitz H. Lumbar stenosis with spondylolisthesis: current concepts of surgical treatment. Clin Orthop Relat Res. 2001;384:54-60. doi:10.1097/00003086-200103000-00008

6. Kornblum MB, Fischgrund JS, Herkowitz HN, Abraham DA, Berkower DL, Ditkoff JS. Degenerative lumbar spondylolisthesis with spinal stenosis: a prospective long-term study comparing fusion and pseudarthrosis. Spine. 2004;29(7):726-734. doi:10.1097/01. BRS.0000119398.22620.92

7. Martin CR, Gruszczynski AT, Braunsfurth HA, Fallatah SM, O'Neil J, Wai EK. The surgical management of degenerative lumbar spondylolisthesis: a systematic review. Spine. 2007;32 (16):1791-1798. doi:10.1097/BRS.0b013e3180bc219e

8. Sengupta DK, Herkowitz HN. Degenerative spondylolisthesis: review of current trends and controversies. Spine. 2005;30(6 Suppl):S71-S81. doi:10.1097/01.brs.0000155579.88537.8e

9. $\mathrm{Kim} \mathrm{R}$, Kim $\mathrm{RH}$, $\mathrm{Kim} \mathrm{CH}$, et al. The incidence and risk factors for lumbar or sciatic scoliosis in lumbar disc herniation and the outcomes after percutaneous endoscopic discectomy. Pain Physician. 2015;18 (6):555-564. doi:10.36076/ppj.2015/18/555

10. Wang HL, Lü FZ, Jiang JY, Ma X, Xia XL, Wang LX. Minimally invasive lumbar interbody fusion via MAST Quadrant retractor versus open surgery: a prospective randomized clinical trial. Chin Med J. 2011;124(23):3868-3874.

11. Haji FA, Cenic A, Crevier L, Murty N, Reddy K. Minimally invasive approach for the resection of spinal neoplasm. Spine. 2011;36(15): E1018-E1026. doi:10.1097/BRS.0b013e31820019f9

12. Yoon SM, Ahn SS, Kim KH, Kim YD, Cho JH, Kim DH. Comparative study of the outcomes of percutaneous endoscopic lumbar discectomy and microscopic lumbar discectomy using the tubular retractor system based on the VAS, ODI, and SF-36. Korean J Spine. 2012;9(3):215-222. doi:10.14245/kjs.2012.9.3.215

13. Li HW, Han X, Wang XF, et al. Curative effect of Quadrant channel transforaminal lumbar interbody fusion in the treatment of recurrent lumbar disc herniation. Clin Med Chin. 2017;33(04):338-341.

14. Huang XC, Liu H. Comparison of minimally invasive fixation and interbody fusion through Quadrant channel with open interbody fusion and internal fixation in the treatment of lumbar disc herniation. Chin J Bone Joint. 2014;3(04):257-260.

15. Li QC, Hu HL, Yan HB, Jin DD. [Unilateral pedicle screw fixation plus interbody fusion by Quadrant system through spatium intermuscular of multifidus]. Zhonghua Wai Ke Za Zhi [Chin J Surg]. 2010;48 (17):1317-1320. [Chinese]. 
16. Xia XL, Wang HL, Lyu FZ, Wang LX, Ma XS, Jiang JY. Mast Quadrant-assisted minimally invasive modified transforaminal lumbar interbody fusion: single incision versus double incision. Chin Med J. 2015;128(7):871-876. doi:10.4103/0366-6999.154280

17. Xiong $\mathrm{C}$, Li T, Kang H, Hu H, Han J, Xu F. Early outcomes of 270-degree spinal canal decompression by using TESSYS-ISEE technique in patients with lumbar spinal stenosis combined with disk herniation. Eur Spine J. 2019;28(1):78-86. doi:10.1007/s00586-0185655-4

18. Nakamura JI, Yoshihara K. Initial clinical outcomes of percutaneous full-endoscopic lumbar discectomy using an interlaminar approach at the L4-L5. Pain Physician. 2017;20(4):E507-E512. doi:10.36076/ ppj.2017.E512

19. Hwang JH, Park WM, Park CW. Contralateral interlaminar keyhole percutaneous endoscopic lumbar surgery in patients with unilateral radiculopathy. World Neurosurg. 2017;101:33-41. doi:10.1016/j. wneu.2017.01.079

20. Middleton SD, Wagner R, Gibson JNA. Multi-level spine endoscopy: a review of available evidence and case report. EFORT Open Rev. 2017;2(7):317-323. doi:10.1302/2058-5241.2.160087

21. Kim HS, Paudel B, Jang JS, et al. Percutaneous full endoscopic bilateral lumbar decompression of spinal stenosis through uniportal-contralateral approach: techniques and preliminary results. World Neurosurg. 2017;103:201-209. doi:10.1016/j.wneu.2017.03.130
22. Wu F, Kong W, Liao W, et al. Percutaneous total endoscopic resection of partial articular processes for treatment of lateral crypt stenosis and lumbar spinal stenosis: technical report and efficacy analysis. Biomed Res Int. 2018;2018:9130182.

23. Wu HK, Zhang LH, Zhang FT, et al. Evaluation of the curative effect of Bies technique under local anesthesia for the treatment of lumbar disc herniation. Contemp Med. 2015;21(36):71-72.

24. Lu Y, Wu ZD, Li ZY, et al. Thirty-five cases of extreme lateral lumbar disc herniation treated with percutaneous lateral foraminal endoscope. Chin J Trad Med Traumatol Orthoped. 2020;28(1):75-81.

25. Manchikanti L, Singh V, Falco FJ, et al. An updated review of automated percutaneous mechanical lumbar discectomy for the contained herniated lumbar disc. Pain Physician. 2013;16(2Suppl): SE151-SE184. doi:10.36076/ppj.2013/16/SE151

26. Li ZZ, Hou SX, Shang WL, Cao Z, Zhao HL. Percutaneous lumbar foraminoplasty and percutaneous endoscopic lumbar decompression for lateral recess stenosis through transforaminal approach: technique notes and 2 years follow-up. Clin Neurol Neurosurg. 2016;143:90-94. doi:10.1016/j.clineuro.2016.02.008

27. Huang TJ, Hsu RW, Li YY, Cheng CC. Less systemic cytokine response in patients following microendoscopic versus open lumbar discectomy. J Orthop Res. 2005;23(2):406-411. doi:10.1016/j. orthres.2004.08.010
International Journal of General Medicine

\section{Publish your work in this journal}

The International Journal of General Medicine is an international, peer-reviewed open-access journal that focuses on general and internal medicine, pathogenesis, epidemiology, diagnosis, monitoring and treatment protocols. The journal is characterized by the rapid reporting of reviews, original research and clinical studies

\section{Dovepress}

across all disease areas. The manuscript management system is completely online and includes a very quick and fair peer-review system, which is all easy to use. Visit http://www.dovepress.com/ testimonials.php to read real quotes from published authors. 